\title{
Retrospective assessment of patterns of recurrence relative to radiotherapy volumes for adjuvant conformal chemoradiotherapy in gastric cancer
}

\author{
Tatiana Conrad $^{1} \cdot$ Steve MacLellan $^{2} \cdot$ Zahra Kassam $^{1} \cdot$ Helen Mackay $^{3}$ • \\ Ida Khalili $^{4} \cdot$ Jenna Sykes $^{5} \cdot$ Tom Purdie $^{1} \cdot$ Allan Okrainec $^{2} \cdot$ Jolie Ringash $^{1}$
}

Received: 11 October 2013/Accepted: 7 August 2015/Published online: 11 September 2015

(c) The International Gastric Cancer Association and The Japanese Gastric Cancer Association 2015

\begin{abstract}
Background Since the Intergroup 0116 study was published in 2000, adjuvant postoperative chemoradiotherapy using CT-planned and 3D conformal/intensity-modulated radiotherapy has been offered routinely to fit patients with resected gastric cancer at Princess Margaret Hospital .The objective of this study was to analyze patterns of disease recurrence with respect to the radiotherapy volumes.

Methods For the date and site (local, locoregional, or distant) of the first recurrence, medical records were reviewed for all patients treated at Princess Margaret Hospital with adjuvant chemoradiotherapy for resected gastric adenocarcinoma (January 1, 2000 to November 30, 2009). Patients whose recurrences were limited to local and/or regional sites were selected for further analysis. Available diagnostic imaging of the recurrence site was registered to the original planning radiotherapy dataset for contouring. If necessary to respect changes in anatomy, the contour was translocated on the basis of anatomic descriptors. The center of mass for each recurrence was
\end{abstract}

Tatiana Conrad

tatiana.conrad@rmp.uhn.on.ca

1 Department of Radiation Oncology, Princess Margaret Cancer Centre, 610 University Avenue, 5th Floor, Toronto, ON M5G 2M9, Canada

2 Department of Surgery, University Health Network, Toronto, Canada

3 Department of Medical Oncology, Princess Margaret Cancer Centre, Toronto, Canada

4 Department of Radiology, University Health Network, Toronto, Canada

5 Department of Statistics, Princess Margaret Cancer Centre, Toronto, Canada identified as a point and its location was categorized according to the isodose encompassing it; in field (90\% or more), marginal (50-89\%), or out of field (less than $50 \%)$.

Results Of all 197 patients, $14(7 \%)$ had isolated locoregional failure, constituting $20 \%$ of all 71 patients with a recurrence. Successful fusions were feasible in five cases. Of these recurrences, four were in field and one was marginal. In a further four cases, visual inspection was used, showing one in-field recurrence, one marginal recurrence, and two out-of-field recurrences. In five patients, either a useable original dataset or diagnostic imaging of the recurrence was not available.

Conclusions The rates of isolated local/locoregional tumor recurrence in this study were low. Of the small number of recurrences available for analysis, most (five of nine) were in field. Further studies involving a larger cohort of patients might allow a more meaningful analysis of trends in the recurrence site with evolving radiotherapy techniques.

Keywords Gastric cancer - Patterns of failure . Chemoradiotherapy - Conformal radiotherapy .

Radiotherapy volumes

\section{Background}

Gastric cancer has traditionally been a surgically managed disease. However, the 5-year overall survival rate with surgery alone is only $15 \%$ for node-positive disease, and the pattern of failure is predominantly locoregional [1-4]. Publication of the Intergroup 0116 trial in 2001 revolutionized the management of gastric adenocarcinoma, with improved overall and disease-free survival in patients 
receiving adjuvant chemoradiotherapy [5]. However, the volume of irradiated tissue used in this trial was large, encompassing the residual stomach and intra-abdominal lymph nodes. Even with technical advances incorporating conformal radiotherapy (CRT), intensity-modulated radiotherapy (IMRT) and image-guided radiotherapy, acute and late toxicity is significant [6].

There have been few articles evaluating patterns of failure in gastric cancer, particularly in patients who have received adjuvant chemoradiotherapy [7-10]. Dikken et al. [9] reported their experience in 2010 and demonstrated a change in recurrence pattern, with distant failures outnumbering locoregional ones. The details of the radiotherapy field design were, however, not provided. In 2004, Lim et al. [10] analyzed failure sites with respect to radiotherapy fields following adjuvant chemoradiotherapy, delivered with a field-based approach, after D2 gastrectomy. In contrast to the report by Dikken et al., the results of Lim et al. demonstrated a high proportion of in-field failures [10]. The purpose of this study was therefore to analyze the patterns of failure in patients with gastric adenocarcinoma following surgical resection and adjuvant chemoradiotherapy with 3D CRT/IMRT delivery and to further categorize the failures in relation to radiotherapy fields.

\section{Methods}

\section{Patients}

A retrospective medical record review of all consecutive patients treated with adjuvant chemoradiotherapy for gastric adenocarcinoma at a single tertiary care cancer center between January 1, 2000 and November 30, 2009 was performed. Tumors were categorized according to the sixth edition of the American Joint Committee on Cancer/Union for International Cancer Control TNM classification . Patients eligible for adjuvant chemoradiotherapy were those who had undergone R0 resection for stage Ib, II, III, or IV (M0) disease, and who had adequate caloric intake (more than $1500 \mathrm{kcal}$ ) and good Eastern Cooperative Oncology Group performance status $(0-2)$. Those with T1N0 and T2N0 tumors without penetration of the muscularis propria were not eligible. The extent of lymph node dissection performed was not documented consistently in the operation notes; therefore, retrieval of 15 or more nodes was used as a surrogate for greater than D1 node dissection as has been previously reported [18, 19]. Adjuvant chemotherapy was delivered according to the protocol used by MacDonald et al. [5] in the Intergroup 0116 trial, or according to the protocol of an institutional phase I/II study delivering infusional 5-fluorouracil and biweekly cisplatin therapy $[5,11]$. Radiotherapy conformed to the INT-0116 protocol, except that it was planned that all patients would undergo CT and all radiotherapy plans were conformal (CRT) or intensity modulated (IMRT).

\section{Radiation plan}

Patients followed a standard diet before simulation and all subsequent radiation fractions, which required them to receive no food or fluids by mouth for $5 \mathrm{~h}$, then to eat the same light meal every day, not less than $1 \mathrm{~h}$ before planning or treatment (one cup of tea, coffee, or other noncarbonated beverage and two slices of bread or toast).

All patients were simulated either in a body-fix fixation device or on a chest board with the arms up, with intravenous contrast medium and $30 \mathrm{ml}$ of water in the stomach. Treatment was planned on the basis of helical CT using 3D CRT or IMRT. The treatment planning approaches evolved during the period of this review. Initially, a junctioned fivefield plan was used (anteroposterior-posteroanterior superiorly, junctioned just above the kidneys to a three-field plan). Later, a nonjunctioned technique was used, with five coplanar fields with or without a noncoplanar field; this general approach then transitioned from 3D CRT to IMRT. For 3D CRT plans, mixed 6-MV/18-MV beams were used, whereas for IMRT plans, only 6-MV beams were used. The target volume included the entire postoperative stomach, anastomoses, regional lymph nodes (right and left gastric, right and left gastroepiploic, celiac, porta hepatis, subpyloric, para-aortic, gastroduodenal, subpancreatic), and the postoperative tumor bed. Inclusion of the splenic hilar nodes (in proximal tumors) and the retropancreaticodoudenal nodes (in distal tumors) was individualized. A 1-cm clinical target volume margin was applied around these regions of interest. The planning target volume margin was determined by the treating radiation oncologist and individualized according to breathing motion measured with either the simulator or 4D CT, with a minimum of $1.5 \mathrm{~cm}$ inferiorly, $1 \mathrm{~cm}$ superiorly, and $1 \mathrm{~cm}$ radially.

\section{Follow-up}

Through the course of concurrent chemoradiotherapy, patients were seen weekly by their treating radiation oncologist and every 4 weeks by their medical oncologist. After completion of the entire adjuvant treatment course, most patients were seen 1 month after treatment, then every 3 months for 2 years, every 6 months until 5 years, and annually thereafter, alternating among their treating specialists. Follow-up CT of the abdomen was normally done 4-12 weeks after treatment, then annually for 5 years. Any clinical suspicion of recurrence resulted in upper gastrointestinal tract endoscopy and/or CT. 


\section{Definition of recurrence}

Diagnosis of recurrence was made by means of diagnostic imaging and/or tissue diagnosis (biopsy or paracentesis), with the time to recurrence defined as the time from diagnosis. Local recurrence was defined as recurrence in the postoperative stomach, anastomoses, duodenal stump, or tumor bed. Regional recurrence was defined as recurrence in a lymph node group specified as a potential radiation target (e.g., splenic hilar and retropancreaticoduodenal nodes were considered regional in both proximal and distal gastric lesions). Distant failure was defined as any lymph node group not specified for radiotherapy, peritoneal carcinomatosis, or recurrence diagnosed by positive fluid cytology findings on paracentesis in the absence of any visible lesion on diagnostic imaging. If the recurrence fell into multiple categories, it was defined as mixed.

\section{Analysis of radiation plans}

Eligible patients were identified with a combined search strategy, involving the institutional cancer registry, the radiotherapy record-and-verify system, and individual clinician files. A retrospective medical record review was undertaken for those cases found in the databases to confirm eligibility. Patients treated during the study period and with disease recurrence were identified. Radiology reports and images were located within or outside (where available) our institution. Recurrence was recorded when one of the two following conditions was met: (1) both the radiology report and dictation by the oncologist indicated recurrence; or (2) dictation indicated a recurrence, and our study radiologist (I.K.) reviewed the imaging and confirmed recurrence. Where radiology reports did not indicate the location on a particular CT slice, the study radiologist reviewed the imaging to confirm the location. For those patients who had recurrence diagnosed only by biopsy or paracentesis, their imaging was reviewed by the study radiologist in an attempt to identify a tissue mass.

Among patients identified as having radiologic evidence of recurrence, we further selected those with isolated local, regional, or locoregional recurrence. For this subset of patients, previous radiotherapy plans were retrieved from our institution's planning systems. Since 2004, we have used the Pinnacle ${ }^{3}$ treatment planning system (version 6.0, Philips Radiation Oncology Systems, Fitchburg, WI, USA). Plans generated on the prior system, CadPlan-Helios version 6.1.5 (Varian), were retrieved when possible; in some cases, they were unavailable for analysis.

In patients for whom diagnostic imaging of the recurrence and the original treatment plans in Pinnacle $^{3}$ were available, rigid registration was performed, fusing the diagnostic dataset with the original treatment plan. Thoracic vertebrae were used for mutual information rigid registration based on the bone in the image within the planning system. It was not technically possible to fuse diagnostic images with CadPlan plans; however, visual inspection was used to transpose the site of recurrence onto original treatment scans. The visual inspection was assessed independently by two radiation oncologists; there were no discrepancies.

On the basis of anatomic landmarks and radiology report descriptions, a volume representing the recurrence was generated on the original treatment dataset and confirmed by the study radiologist. For patients with local and locoregional recurrences, the center of mass of the recurrent lesion was identified and used as a representative point. Such points were analyzed according to their relation to the isodose lines on the original treatment plans. Recurrences were considered to be in field if they were encompassed by the $90 \%$ isodose line, marginal if they were between the 50 and $90 \%$ isodose lines. and out of field if they were not covered by the $50 \%$ isodose line.

\section{Statistical analysis}

Fisher's exact test was used to compare whether there were any differences in the type of recurrence (no recurrence, locoregional recurrence, or distant and locoregional recurrence) and disease stage, $\mathrm{T}$ category, $\mathrm{N}$ category, or sex. To determine whether there was any difference between the type of recurrence and age, the Kruskal-Wallis test was used. The Kaplan-Meier method was used to estimate the median time to recurrence for all patients. All statistical analyses were performed in $\mathrm{R}$ version 2.12.1 [11]. Two-sided $P$ values of less than 0.05 were used to assess statistical significance.

\section{Results}

From January 1, 2000 to November 30, 2009, 197 patients were identified. Demographic and disease-related features are shown in Table 1. Most patients presented with American Joint Committee on Cancer/Union for International Cancer Control TNM classification (sixth edition) stage II $(33 \%)$ or stage III (40\%) disease. All underwent $\mathrm{R} 0$ resection. The median number of lymph nodes retrieved was 16 . More than half the patients $(55.3 \%)$ had greater than D1 resection. Median follow-up was 28.7 months (range 4.0-99.4 months).

MacLellan et al. [12, 13] have reported on recurrence outcomes. Of the 197 patients treated, 71 recurrences (36\%) were identified, of which $57(80 \%)$ were classified as having distant metastases as either the only site of failure 
Table 1 Demographic and pathologic comparison of patients

\begin{tabular}{|c|c|c|c|c|c|}
\hline Characteristic & $\begin{array}{l}\text { All patients } \\
(N=197)\end{array}$ & $\begin{array}{l}\text { No recurrence } \\
(N=126)\end{array}$ & $\begin{array}{l}\text { Locoregional recurrence } \\
(N=14)\end{array}$ & $\begin{array}{l}\text { Distant and locoregional } \\
\text { recurrence }(N=57)\end{array}$ & $P^{\mathrm{b}}$ \\
\hline \multicolumn{6}{|l|}{ Age $(\text { years })^{\mathrm{a}}$} \\
\hline Median & 58 & 59 & 55 & 58 & 0.14 \\
\hline Range & $22-80$ & $22-78$ & $32-68$ & $26-80$ & 0.14 \\
\hline Female sex & $73(37 \%)$ & $46(37 \%)$ & $4(29 \%)$ & $23(40 \%)$ & 0.70 \\
\hline $\mathrm{T}$ category & & & & & 0.00046 \\
\hline 1 & $20(10 \%)$ & $20(16 \%)$ & $0(0 \%)$ & $0(0 \%)$ & \\
\hline 2 & $76(39 \%)$ & $54(43 \%)$ & $4(29 \%)$ & $18(32 \%)$ & \\
\hline 3 & $94(48 \%)$ & $49(39 \%)$ & $10(71 \%)$ & $35(61 \%)$ & \\
\hline 4 & $7(4 \%)$ & $3(2 \%)$ & $0(0 \%)$ & $4(7 \%)$ & \\
\hline $\mathrm{N}$ category & & & & & 0.06 \\
\hline 0 & $36(18 \%)$ & $24(19 \%)$ & $4(29 \%)$ & $8(14 \%)$ & \\
\hline 1 & $114(58 \%)$ & $78(62 \%)$ & $4(29 \%)$ & $32(56 \%)$ & \\
\hline 2 & $33(17 \%)$ & $19(15 \%)$ & $4(29 \%)$ & $10(18 \%)$ & \\
\hline 3 & $14(7 \%)$ & $5(4 \%)$ & $2(14 \%)$ & $7(12 \%)$ & \\
\hline Stage & & & & & 0.0003 \\
\hline $\mathrm{Ib}$ & $37(19 \%)$ & $34(27 \%)$ & $1(7 \%)$ & $2(4 \%)$ & \\
\hline II & $65(33 \%)$ & $43(34 \%)$ & $5(36 \%)$ & $17(30 \%)$ & \\
\hline III & $78(40 \%)$ & $43(34 \%)$ & $6(43 \%)$ & $29(51 \%)$ & \\
\hline IV & $17(9 \%)$ & $6(5 \%)$ & $2(14 \%)$ & $9(16 \%)$ & \\
\hline
\end{tabular}

${ }^{\text {a }}$ Age at diagnosis

b Tests for a difference in recurrence type (none vs locoregional vs distant and locoregional). The Kruskal-Wallis test was used for age; Fisher's exact test was used for categorical variables (sex, T category, $\mathrm{N}$ category, and stage.

or as a component of failure. However, on radiology review as performed for this subsequent analysis, one further patient initially classified as having isolated locoregional failure was found to also have distant metastases, for a total of 63 (84\% of all recurrences). The median time to recurrence was 6.8 years (95\% confidence interval, 3.8 to not reached).

Of all 197 patients, 14 (7\%) had isolated locoregional failure, constituting $20 \%$ of all 71 patients with recurrence (Fig. 1). Original plans, as well as cross-sectional imaging of the recurrence, were sought for all cases. In five patients, either a useable original dataset or diagnostic imaging of recurrence was not available. Successful fusions were feasible in five cases (Fig. 2). Of these recurrences, four were in field (Fig. 3) and one was marginal. Visual inspection was used to localize recurrences in four cases, three CadPlan cases (one recurrence in field, one marginal, and one out of field), and one case planned in Pinnacle ${ }^{3}$ but for which the patient's body habitus had changed so significantly that attempts to appropriately fuse the planning and recurrence CT images were deemed to be too inaccurate (classified as out-of-field recurrence). Overall, among the 14 isolated locoregional failures in our series, five were in field, two were marginal, two were out of field, and five were unclassifiable.

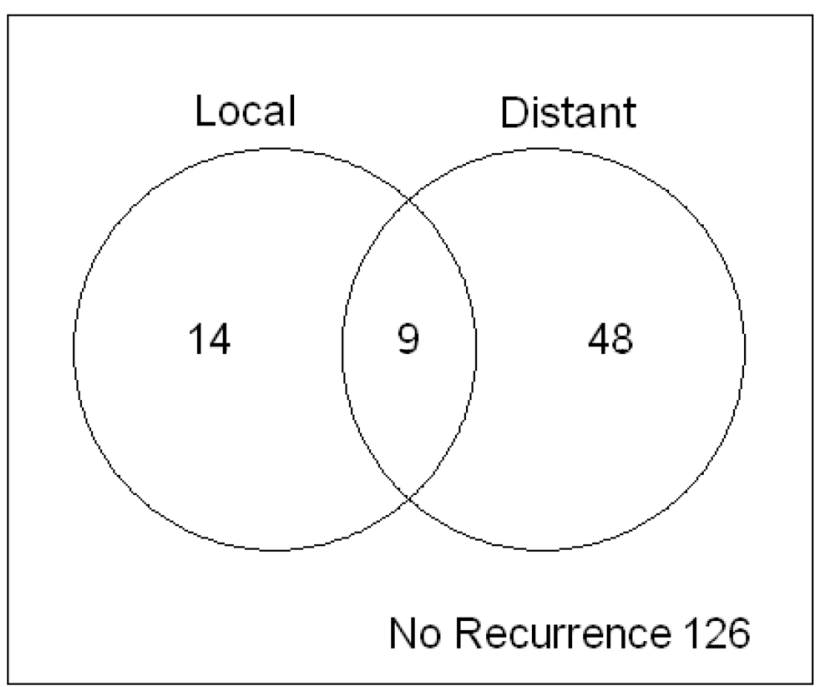

Fig. 1 Failure patterns

\section{Discussion}

Since the adoption of the Intergroup 0116 protocol for treatment of resected gastric adenocarcinoma, studies from both the eastern hemisphere and the western hemisphere 
Fig. 2 CONSORT diagram demonstrating breakdown of patients by recurrence status

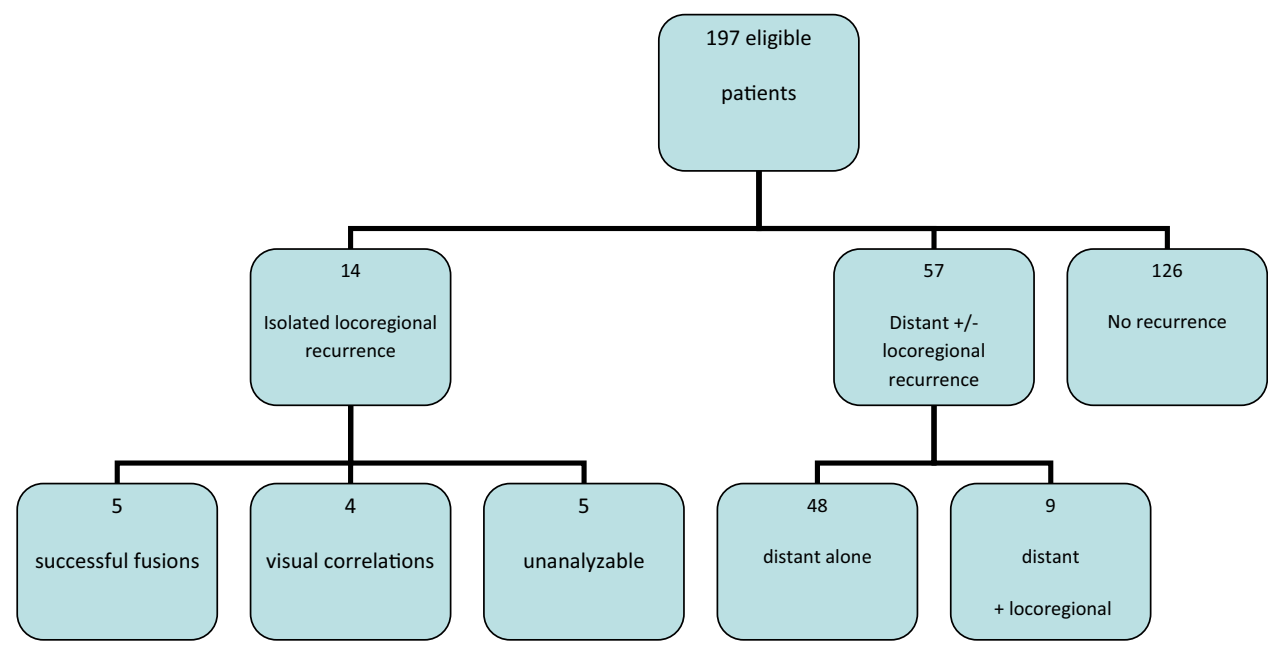

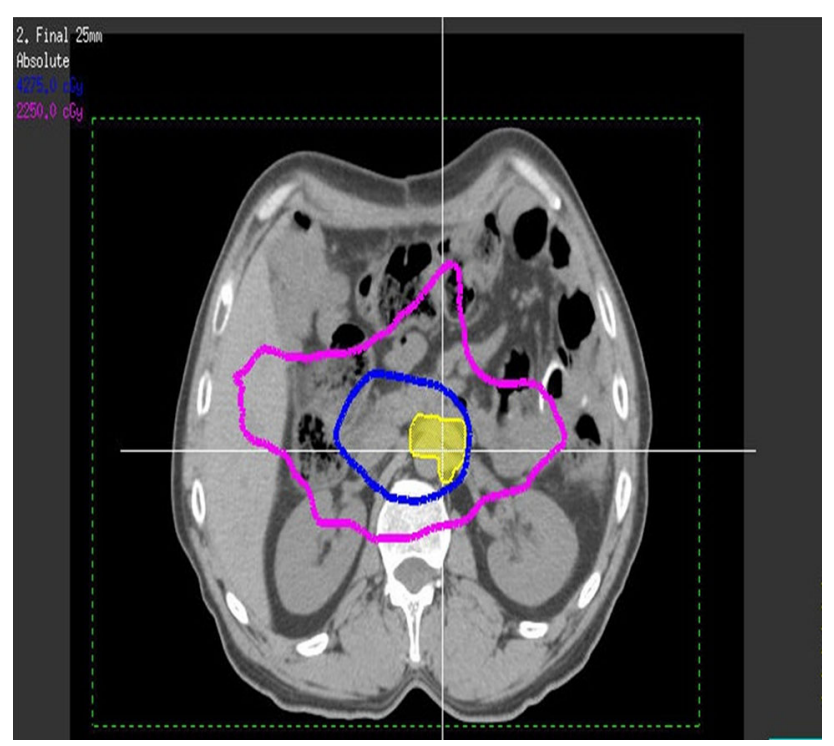

Fig. 3 Radiotherapy isodose curves with local recurrence. Radiotherapy plan demonstrating the $95 \%$ (4275 cGy) and $50 \%$ (2250 cGy) isodose lines in blue and purple, respectively, with the recurrence volume contoured in yellow, highlighting an in-field recurrence

have confirmed improved overall survival and disease-free survival as compared with surgical resection alone. Furthermore, studies have demonstrated a shift in the primary relapse pattern from locoregional to distant recurrence. Published data on the rates and patterns of recurrence following extensive surgery have been varied, and this is likely attributable to selection in patient cohorts, the time points at which recurrences were identified, and the method by which recurrences were classified. The landmark article by Gunderson and Sosin [2] from 1982 demonstrated that most failures following curative resection for gastric adenocarcinoma were locoregional, accounting for $22.9 \%$ of isolated failures and as a component of failure in up to
$68.6 \%$ of patients. On the basis of these findings, they provided recommendations about the potential utility of adjuvant radiotherapy and chemotherapy, and suggested treatment fields. In a retrospective analysis, D'Angelica et al. [7] reported a locoregional relapse rate of $17 \%$, with no difference observed in those receiving D2 or greater surgery. The Dutch Gastric Cancer Trial group, however, has observed lower locoregional recurrence rates with D2 surgery $(25 \%)$ than with D1 surgery (41\%) [20]. Since adjuvant chemoradiotherapy was adopted, Lim et al. [10] have analyzed 114 patients with failure among a cohort of 291 patients treated with traditional parallel opposed fields and found isolated locoregional failure in 13 patients (4\% of cases; $11 \%$ of failures). They also examined the frequency of local and/or regional sites of failure among all sites of failure; in these 114 patients, there were 156 sites of failure, of which $55(19 \%)$ were locoregional. However, in that study, "local or regional failure" was defined as failure within radiotherapy fields, so the results cannot be compared directly with our analysis. There have been no further attempts to categorize sites of recurrence relative to the radiation fields used today.

Current radiotherapy techniques with 3D CRT and IMRT permit tailoring of the irradiated volume to avoid adjacent critical structures, such as the kidneys and liver $[14,15]$. However, to use such technology optimally, it is even more important to evaluate the sites of recurrence, and to hypothesize about rational tailoring of the radiotherapy to minimize both locoregional recurrence and late adverse events.

What could such analyses teach us? Out-of-field recurrences might identify new targets to be included in adjuvant radiotherapy volumes. Primarily marginal recurrence could suggest the need for more generous clinical target volume or planning target volume margins, or could even argue against the use of highly conformal techniques, favoring 
traditional field-based radiotherapy with less risk of a geographic miss. A primary pattern of in-field recurrence might lead to consideration of the role of dose escalation. Although limited clinical data have been published on this subject, several small studies have demonstrated that dose escalation with intraoperative radiotherapy might be feasible without excessive normal tissue toxicity [16].

Our study confirmed a dramatic change in the pattern of recurrence as compared with surgery alone. The great majority $(83 \%)$ of recurrences observed involved distant disease sites. Consequently, the isolated locoregional failure rate among our 197 cases was only $7 \%$, at least a $10 \%$ improvement in comparison with most conservative data published on recurrence following surgery alone [7]. Our data suggest the very significant benefit of radiotherapy, but our ability to learn from the handful of patients with isolated locoregional failure is limited. Our study was further limited by challenges in obtaining outside imaging at the time of recurrence, and problems accessing older plans in an obsolete planning system.

Another limitation of our method was that some patients had experienced a significant change in body habitus subsequent to their original treatment. This resulted in less certainty about the correlation of normal soft tissue anatomy between treatment plan imaging and images documenting recurrence. Although deformable registration methods could potentially help to address these concerns, they are still considered experimental and were not performed in this analysis.

This work illustrates the difficulties inherent in studying infrequent and late-occurring events, which are similar to those which occur in the study of late radiation toxicity or the occurrence of radiation-generated second primary cancers. The very low rate demonstrated in our study means that single-institution studies, even from large institutions such as ours, will not generate sufficient numbers of patients to study such uncommon events. However, the very long-term follow-up required can be prohibitive in a multicenter, cooperative group trial such as the Intergroup 0116 trial. One potential strategy may be to use the cooperative group infrastructure to set up multicenter registries specific to certain diagnoses and treatments, such as gastric cancer adjuvant chemoradiotherapy, that would track patients with locoregional recurrence, late toxicity, or second primary cancers. A similar initiative by the Pediatric Oncology Group of Ontario allows the monitoring of patient outcomes and long-term effects of childhood cancer and its treatment. This pooled resource system has allowed better understanding of the impact of treatment of childhood malignancies [17].

Of the nine patients with locoregional recurrence that we were able to analyze, five (56\%) had in-field recurrence, two (22\%) had marginal recurrence, and two had out-of- field recurrence. Although this is overall relatively reassuring regarding the safety of highly conformal radiotherapy techniques, attention to careful contouring, assessment of organ motion, and daily set up remains essential. Moreover, given the small sample size, the confidence associated with these estimates is low. What this study does demonstrate, however, is the feasibility of fusing recurrence CT datasets with initial plans, and the potential role of multicenter collaboration in assessment of sites of recurrence relative to radiotherapy volumes.

\section{Conclusions}

The rates of isolated local/locoregional tumor recurrence in this study were low. Of the small number of locoregional recurrences available for analysis, most were either in field or marginal. Further studies involving a larger cohort of patients might allow a more meaningful analysis of trends in the recurrence site with evolving radiotherapy techniques.

\section{References}

1. Landry J, Tepper JE, Wood WC, Moulton EQ, Koerner F, Sullinger J. Patterns of failure following curative resection of gastric carcinoma. Int J Radiat Oncol Biol Phys. 1990;19:1357-62.

2. Gunderson LL, Sosin H. Adenocarcinoma of the stomach: areas of failure in a re-operation series (second or symptomatic look) clinicopathologic correlation and implications for adjuvant therapy. Int J Radiat Oncol Biol Phys. 1982;8:1-11.

3. Yoo CH, Noh SH, Shin DW, et al. Recurrence following curative resection for gastric carcinoma. Br J Surg. 2000;87:236-42.

4. Hundahl SA, Menck HR, Mansour EG, Winchester DP. The national cancer data base report on gastric carcinoma. Cancer. 1997;80(12):2333-41.

5. MacDonald JS, Smalley SR, Benedetti J, et al. Chemoradiotherapy after surgery compared with surgery alone for adenocarcinoma of the stomach or gastroesophageal junction. N Engl J Med. 2001;345:725-30.

6. Kassam Z, Lockwood G, O'Brien C, et al. Conformal radiotherapy in the adjuvant treatment of gastric cancer: review of 82 cases. Int J Radiat Oncol Biol Phys. 2006;65(3):713-9.

7. D'Angelica M, Gonen M, Brennan MF, et al. Patterns of initial recurrence in completely resected gastric adenocarcinoma. Ann Surg. 2004;240:808-16.

8. Reed VK, Krishnan S, Mansfield PF, et al. Incidence, natural history, and patterns of locoregional recurrence in gastric cancer patients treated with preoperative chemoradiotherapy. Int $\mathbf{J}$ Radiat Oncol Biol Phys. 2008;71(3):741-7.

9. Dikken JL, Jansen EP, Cats A, et al. Impact of the extent of surgery and postoperative chemoradiotherapy on recurrence patterns in gastric cancer. J Clin Oncol. 2010;28(14):2430-6.

10. Lim DH, Kim DY, Kang MK, et al. Patterns of failure in gastric carcinoma after D2 gastrectomy and chemoradiotherapy: a radiation oncologist's view. Br J Cancer. 2004;91(1):11-7.

11. R Development Core Team. R: a language and environment for statistical computing. Vienna: R Foundation for Statistical Computing; 2010. 
12. Kassam Z, MacKay H, Buckley CA, et al. Adjuvant chemoradiation for gastric cancer with infusional 5-fluorouracil and cisplatin: a phase I study. Curr Oncol. 2010;17(4):34-4112.

13. MacLellan SK, MacKay HJ, Ringash J, et al. Laparoscopic gastrectomy for patients with advanced gastric cancer produces oncologic outcomes similar to those for open resection. Surg Endosc. 2012. doi:10.1007/s00464-011-2118-y.

14. Ringash J, Khaksar SJ, Oza A, et al. Post-operative radiochemotherapy for gastric cancer: adoption and adaptation. Clin Oncol. 2005;17:91-5.

15. Callister MD, Gunderson LL. Advancements in radiation technique for gastric cancer. J Natl Compr Canc Netw. 2010;8(4): 428-35.

16. Fu S, Lu JJ, Zhang Q, et al. Intraoperative radiotherapy combined with adjuvant chemoradiotherapy for locally advanced gastric adenocarcinoma. Int J Radiat Oncol Biol Phys. 2008;72(5): 1488-94.

17. Pediatric Oncology Group of Ontario. POGONIS-childhood cancer database. http://www.pogo.ca/pogonis/ (2015).

18. Helyer LK, O'Brien C, Coburn NG, et al. Surgeon's knowledge of quality indicators for gastric cancer surgery. Gastric Cancer. 2007;10:205-14.

19. Karpeh MS, Leon L, Klimstra D, et al. Lymph node staging in gastric cancer: is location more important than number? An analysis of 1038 patients. Ann Surg. 2000;232:362-71.

20. Songun I, Puter H, Kranenbarg EM, et al. Surgical treatment of gastric cancer: 15-year follow-up results of the randiomised nationwide Dutch D1D2 trial. Lancet Oncol. 2010;11:439-49. 